\title{
Structural and functional characterization of transcription factor binding sites: from bioinformatics to hormone biosensors
}

Dolgikh V. ${ }^{1}$, Levitsky V. ${ }^{1,2}$, Oshchepkov D. ${ }^{1}$, Zemlyanskaya E. ${ }^{1,2 *}$

${ }^{1}$ Institute of Cytology and Genetics, SB RAS, Novosibirsk, Russia

${ }^{2}$ Novosibirsk State University, Novosibirsk, Russia

*email: ezemlyanskaya@bionet.nsc.ru

ETHYLENE INSENSITIVE 3 (EIN3) transcription factor is the master regulator of gene expression in response to plant hormone ethylene. It binds a short nucleotide sequence referred to as EBS to induce transcription. Arabidopsis thaliana reporter EBS:GUS driven by such EIN3 binding site is widely used as a sensor for detection of ethylene signaling. However, the role of EBS arrangement (syntax) in EIN3 functioning was not investigated so far. Here we accomplish a systematic bioinformatics analysis of EIN3 bound sequences in Arabidopsis genome to shed light on molecular mechanisms utilized for regulation of transcriptional response to ethylene in plants.

Integarating publicly available ChIP-seq and DAP-seq data on EIN3 binding with RNAseq data on ethylene-induced transcriptomes in Arabidopsis seedlings we discovered a previously unknown EBS architecture that is enriched in EIN3 bound sequences to a much greater extent than a single EBS motif. This new configuration is a head-to-head inverted repeat of EBS-like sequences with 1 bp overlap referred to as $2 \mathrm{EBS}(-1)$. We also demonstrated that the inverted repeat of the coreEBS with the overlap of the motifs but not with a spacer is enriched in the Arabidopsis genome. Based on these findings we consider that 2EBS(-1) is a canonical EIN3 binding site in A. thailana genome. We further showed that of all EBS configurations under study only 2EBS(-1) was significantly associated with transcriptional response of EIN3 targets to ethylene treatment. Moreover, it tended to cause a more pronounced transcriptional response than other EBS configurations. We used these findings to design a new genetic sensor for highly sensitive detection of ethylene signaling. Taken together, this work provides new insight on the molecular mechanisms utilized for regulation of transcriptional response to ethylene in plants.

Acknowledgments: This work was supported by the RSF grant No. 20-14-00140. 